\title{
Experimental Study of Synchro-Betatron Coupling Induced by Dipole Modulation
}

\author{
M. Syphers,1 M. Ball,2 B. Brabson,2 J. Budnick,2 D. D. Caussyn,2 A. W. Chao,1 J. Collins, 2 \\ V. Derenchuk, ${ }^{2}$ S. Dutt, ${ }^{1}$ G. East,2 M. Ellison, ${ }^{2}$ T. Ellison, ${ }^{2}$ D. Friesel, ${ }^{2}$ W. Gabella, ${ }^{4}$ B. Hamilton, ${ }^{2}$ \\ H. Huang,2 W. P. Jones, ${ }^{2}$ S. Y. Lee, ${ }^{2}$ D. Li, ${ }^{2}$ M. G. Minty, ${ }^{3}$ S. Nagaitsev, ${ }^{2}$ K. Y. Ng, ${ }^{4}$ X. Pei, ${ }^{2}$ \\ G. Rondeau, ${ }^{2}$ T. Sloan, 2 L. Teng, 5 S. Tepikian,6 Y. Wang, 2 Y. T. Yan, 1 and P. L. Zhang 1 \\ 1 The SSG Laboratory, 2550 Beckleymeade Avenue, Dallas, Texas 75237-3946 \\ 2 Indiana University Cyclotron Facility, Indiana University, Bloomington, Indiana 47405 \\ ${ }^{3}$ Stanford Linear Accelerator Center, MS26, Box 4349, Stanford, California 94309 \\ ${ }^{4}$ Fermilab, P.O. Box 500, Batavia, Illinois 60510 \\ ${ }^{5}$ Aryonne National Laboratory, APS, 9700 South Cass Avenue, Illinois 60439 \\ ${ }^{6}$ Brookhaven National Laboratory, Upton, New York 11973
}

(Received 22 March 1993)

\begin{abstract}
Synchro-betatron coupling in a proton storage ring with electron cooling was studied experimentally by modulating a transverse dipole field close to the synchrotron frequency. The combination of the electron cooling and transverse field modulation on the synchrotron oscillation is equivalent to a dissipative parametric resonant system. The proton bunch was observed to split longitudinally into two pieces, or beamlets, converging toward attractors of the dissipative system. These phenomena might be important in understanding the effect of ground vibration on the Superconducting Super Collider beam, and the effect of power supply ripple on the Relativistic Heavy-Ion Collider beam.
\end{abstract}

PACS numbers: 41.85.-p, 03.20.+i, 05.45.+b, 29.20.Dh

In a recent experiment [1], we found that the longitudinal response of a beam to the phase modulation of the accelerating field exhibited characteristics of a parametric resonant system [2]. The equation of motion for phase oscillations [3] of a particle in a synchrotron, in the absence of forced oscillations, is given by

$$
\mathbb{C}+\mathrm{w} ;(\sin 4>-\sin 4>0)=0 .
$$

Here $\left.w_{8}=w_{0} \quad ; ; \mathrm{J}\right]$ is the small amplitude synchrotron angular frequency at $4>_{0}=0$, wo is the angular revolution frequency, and $h, V$, and $\Phi_{0}$ are, respectively, the harmonic number, the peak rf voltage, and the synchronous phase angle, ( $3 c$ and $E$ are, respectively, the speed and the energy of the particle, and $r J$ is the phase slip factor. For this experiment, we have $h=1, r J \ldots . . . \cdots-0.86, \Phi_{0}=0$, and $f_{0}=1.03168 \mathrm{MHz}$ for protons with kinetic energy of $45 \mathrm{MeV}$. Since the synchrotron frequency depends on the rf voltage, we chose an rf voltage of $41 \mathrm{~V}$ to obtain a synchrotron frequency of $262 \mathrm{~Hz}$ in order to avoid harmonics of the $60 \mathrm{~Hz}$ ripple on magnet supplies. The synchrotron tune is given by vsyn $==\quad 2.54 \times 10-4$. Transversely, the horizontal and vertical deviations from the closed orbit of a beam particle satisfy Hill's equation [4]:

$$
\frac{d^{2} x}{d s^{2}}+K_{x}(s) x=\frac{\Delta B_{z}}{B \rho}, \quad \frac{d^{2} z}{d s^{2}}+K_{z}(s) z=-\frac{\Delta B_{x}}{B \rho} .
$$

Here $K x(s), K z(s)$ are focusing functions due to quadrupoles, $B p=\bar{z}$ is the magnetic rigidity, $s$ is the longitudinal particle coordinate and $6 . . B x, 6 . . B z$ are lin- ear or nonlinear error magnetic fields. In the linear approximation, the error-free transverse Hill's equation can be solved to obtain, $y=-J \overline{2(3 y(s) J y} \cos [</>y(s)+X y]$, $\langle>y(s)=J$; : , where $y$ stands for either $x$ or $z$. Thus particles are executing betatron oscillations transversely about the closed orbit of an accelerator. Here $(3 y(s)$ is the betatron amplitude function, $J y$ is the invariant action, $\langle/>y(s)$ is the betatron phase, and $X y$ is an initial phase angle. The numbers of betatron oscillations in one revolution are called betatron tunes given by $v_{y}={ }_{2} f:$. We chose $V x=3.828, V z=4.858$ for this experiment to avoid nonlinear betatron resonances. The corresponding fractional part of the horizontal betatron frequency was $(4-V x) f o=177 \mathrm{kHz}$.

Coupled motion between the transverse and longitudinal degrees of freedom is called synchro-betatron (SB) coupling. The SB coupling is important to electron storage rings [5], where the fractional parts of the synchrotron and betatron tunes are of the same order of magnitude. On the other hand, the fractional part of the betatron and synchrotron tunes differ substantially in proton storage rings, and the coupling between longitudinal and transverse motions is less important. To the knowledge of the authors, SB coupling has not been observed previously in proton storage rings. For the SSC, where the synchrotron frequency varies from $7 \mathrm{~Hz}$ at injection energy to $4 \mathrm{~Hz}$ at $20 \mathrm{TeV}$, SB coupling may arise due to ground vibration. At Relativistic Heavy Ion Collider, the synchrotron frequency ramps through $60 \mathrm{~Hz}$ around $17 \mathrm{GeV} / c$ for heavy ion beams, SB coupling may result from power supply ripple.

The dominant effect of ground vibration or power supply ripple is a modulation of the dipole field. A slow adiabatic dipole modulation gives rise to a closed orbit modulation. Provided that the resulting closed or- 
bit modulation is much smaller than the beam width, the adiabatic modulation usually does not limit the performance of colliders or storage rings. A considerable amount of detailed analysis has been reported and compiled [6]. However, performance degradation can arise from nonadiabatic modulations, which occur naturally at resonance conditions, e.g., transverse modulation at the synchrotron frequency. A detailed study of this issue is therefore needed. In this paper, we present experimental data, taken at the Cooler synchrotron at the Indiana University Cyclotron Facility, for transverse modulations producing SB coupling, similar to what may arise from ground vibrations or power supply ripples.

The experimental procedure started with a single bunch of about $5 \times 10^{8}$ protons with kinetic energy of $45 \mathrm{MeV}$. The cycle time was $11 \mathrm{~s}$. The injected beam was electron-cooled and was simultaneously modulated by a small dipole. A 23 em window frame ferrite dipole magnet was used to produce the transverse dipole modulation. The effective length of the dipole was measured to be $£=0.27 \mathrm{~m}$. The horizontal dispersion function Dx was about $4.0 \mathrm{~m}$ at the modulation dipole location. At the end of $7 \mathrm{~s}$, the beam-centroid displacements were measured, digitized, and recorded from signals of beam position monitors (BPM). The longitudinal phase was measured by comparing the time difference between the BPM sum signal and the signal from an rf clock. The beam position at a high dispersion location was used to measure the momentum deviation, which is related to the off momentum closed orbit by $X c 0=\boldsymbol{D} \times$ with $D x, \ldots . \cdots, 3.9$ m. A total of 8 channels were used to obtain 6D phase space maps with 16384 points recorded at 10 turn intervals. Details of our detection system had been reported earlier $[1,7]$.

With a horizontal dipole (vertical field) modulation at location so, the horizontal closed orbit becomes [4],

$X \operatorname{co}(t)=y^{\prime} \mathrm{xs} \quad$ so) $B(t) \cos [\mathrm{rrvx}-1</>x(\mathrm{~s})-</>x(\mathrm{so}) l]$, where $B(t)=B B) £=8$ sinwmt with $8=8$;,£. Furthermore, if the dispersion function at the modulating dipole location is not zero, the path length is also modulated. The change of the circumference is given by $\square=D x B(t)$. The corresponding rf phase difference becomes, $\langle>>=2 \operatorname{rrh} \boldsymbol{g}$, where $\boldsymbol{C}=86.8224 \mathrm{~m}$ is the circumference of the IUCF Cooler Ring. In our experiment, the maximum rf phase shift per turn is

$=0.78 \times \mathrm{W}-5 \mathrm{Bm}[\mathrm{G}]$ radians. The longitudinal phase space coordinates, $(\langle/\rangle$, ), at the nth and the $(n+1)$ th revolutions are transformed according to the following mapping equations [4]:

$$
\begin{gathered}
<>n+1=\langle>n+2 \operatorname{rrhry}<;>n+<>, \\
(P)_{n+1}=(P)_{n}+-\sin <>>n+1-A(P)_{n}^{\prime}
\end{gathered}
$$

where the fractional momentum deviation of a particle,
( $>$ is the conjugate variable to the synchrotron phase angle \langle\rangle , and $A$ is the phase space damping parameter related to the electron cooling. Thus the synchrotron equation of motion, in the presence of transverse modulation, becomes

$$
\$+2 a t P+w \sin </>=W m W_{s} a \cos w m t+2 a w_{8} a \sin w m t,
$$

with the damping coefficient $\boldsymbol{a}=\boldsymbol{A}$. With an electron current of $0.75 \mathrm{~A}$, the damping time for the $45 \mathrm{MeV}$ protons was measured to be about 0.4 sora $=2.5 \mathrm{~s}-\mathrm{I}$, which was indeed small compared with $w_{8}=1646 \mathrm{~s}-1$.

Because the synchrotron frequency is much smaller than the revolution frequency in proton storage rings, the phase errors for each turn accumulate. The phase modulation amplitude is enhanced by the factor 2 ., i.e., the effective phase modulation amplitude parameter [1], $a$, is given by

$$
a=\frac{h w o D x 8}{w_{8} C}=\underset{27 \mathrm{rws}}{\mathbf{\Phi}} \text { - }
$$

Even though the cooling was weak, sufficient time was allowed before making measurements that the transient solution of Eq. (2) was damped out. We therefore measured the steady state solution, in contrast to our earlier experiment [1] where the transient solutions of Eq. (2) were measured. Let the stationary solution of the nonlinear parametric dissipative resonant system be given by \langle\rangle$\rangle, \ldots ., \cdot g \sin (w m t-x)$, where we have used a single harmonic approximation. Expanding the term $\sin <>>$ in Eq. (2) up to the first harmonic, we obtain the equation for the modulation amplitude $g$ as

$\left[-w ; g+2 w J l\left(Y W+[2 \mathrm{awmg}]^{2}=\left[w m w_{s} a\right]^{2}+[2 \mathrm{aw}, \mathrm{a}]^{2}\right.\right.$,

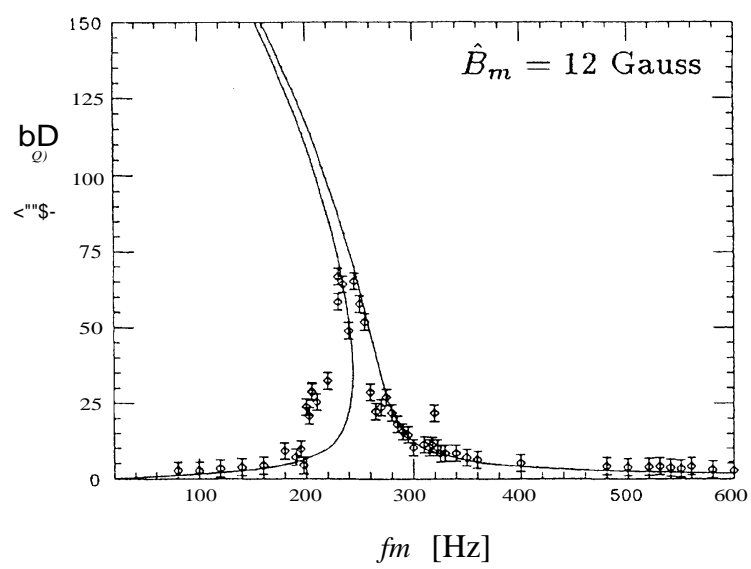

FIG. 1. The phase amplitude response measured from a BPM as a function of the modulation frequency around the synchrotron tune. At Ern $=12 \mathrm{G}$, the $60 \mathrm{~Hz}$ power supply ripple generated structure at 320 and $200 \mathrm{~Hz}$. Solid lines correspond to attractor solutions calculated with the parameters $a$ of Eq. (3). Here, $J>g$ in the text. 
where $\mathbf{J}_{\mathbf{1}}$ is the Bessel function [8] of order 1 . Figure $\mathbf{1}$ shows the longitudinal phase amplitude, measured with the phase detector (diamonds), as a function of sinusoidal transverse modulation frequencies for the modulation amplitude with $\mathrm{Bm}=12 \mathrm{G}$. The amplitudes of the steady state solutions of Eq. (4) were also shown in Fig. 1 with $0:=2.5 \mathrm{~s}-1$ and the parameter $a$ obtained from Eq. (3). Note here that there are multiple solutions of Eq. (4) when the modulation frequency is below the critical bifurcation frequency [1], we

Existence of bifurcation solutions of Eq. (2) had previously been verified $[1,2]$. Steady state solutions of Eq. (4) are also attractors of the dissipative dynamics system. The existence of a unique phase factor $X$ for the solutions of the dissipative parametric resonant equation implies that the attractor is a single phase space point rotating with modulation frequency $\mathbf{W m}$. Figure 2 shows the measured longitudinal beam profile in time domain integrated over many synchrotron periods, as shown on an oscilloscope triggered at the rf frequency, where a single oscilloscope trace corresponds to the projection of the longitudinal charge density as the beam passes through the BPM. The modulation amplitude $\mathrm{Bm}$ was $4 \mathrm{G}$, and the modulation frequencies were 210,220 , and $230 \mathrm{~Hz}$ in the left column and 240, 250, and $260 \mathrm{~Hz}$ in the right column. Using a fast sampling oscilloscope (HP54510A)
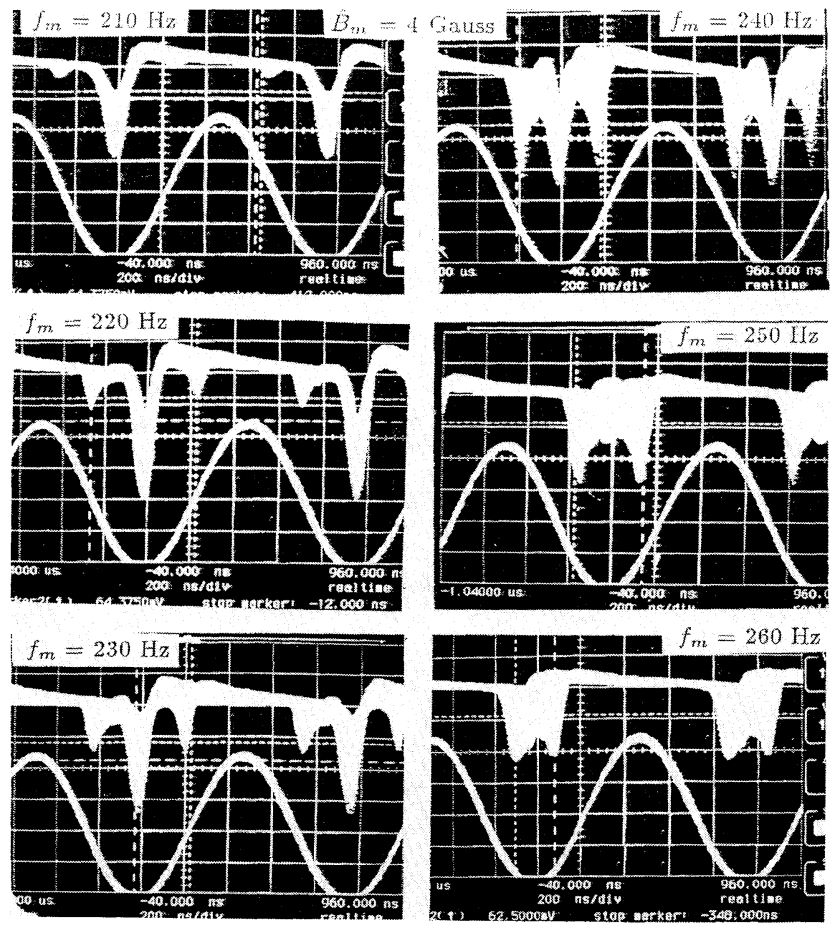

FIG. 2. The real time longitudinal beam profile, observed from an oscilloscope, shows the splitting of beam bunch into beamlets below the bifurcation frequency. The modulation amplitude was $E m=4 \mathrm{G}$. The sine waves in this figure are the rf wave form. for a single trace, the bunch was indeed observed to split into two beamlets located at amplitudes corresponding to the steady state solutions of this dissipative parametric system. Both beamlets rotate in the synchrotron phase space at the modulating frequency, measured from the FFT of the phase signal. The two outer peaks correspond to an elongated outer beamlet at the upright position so that the peak current is large. When the beamlet rotates to the central position in the phase coordinate, the bunch is extended longitudinally with a smaller peak current. The aspect ratio of these two beamlets can be obtained by fitting the observed profile shown in Fig. 2. For example, the aspect ratio of the outer beamlet at $220 \mathrm{~Hz}$ was found to be about 1:3 from the profile of Fig. 2. The peak current is therefore reduced by a factor of 3 at the center of the phase coordinate. As the modulating frequency is increased towards the synchrotron frequency, the outer peak moves in and its population intensity increases. When the modulating frequency was
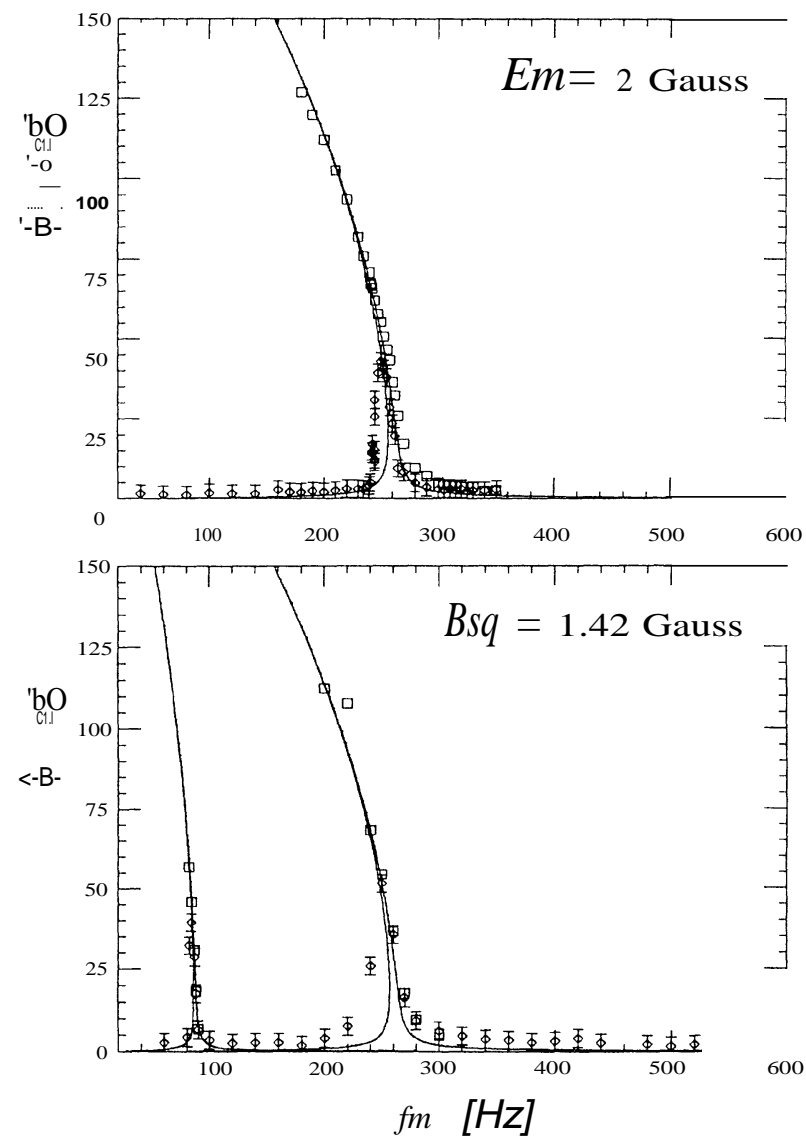

FIG. 3. The phase amplitude of the outer beamlet measured from a fast sampling oscilloscope and the phase amplitude measured from a wall gap monitor as functions of modulation frequency for the sinusoidal wave with $E m=2$ $\mathrm{G}$ and the square wave with $E s q=1.42 \mathrm{G}$. For the square wave modulation, a large amplitude response was expected and observed at $3 w m=w \bullet$. Again, $\mathbb{\$}=g$. 


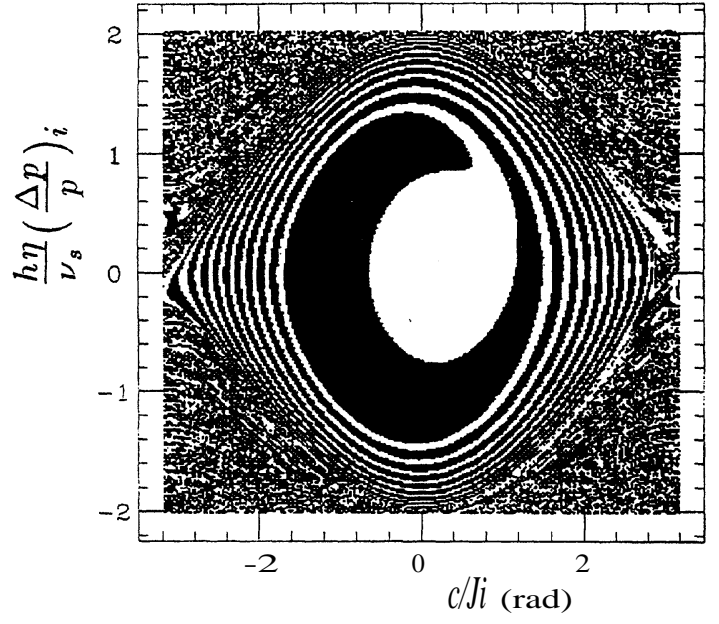

FIG. 4. Initial phase space coordinates, obtained from a numerical simulation of Eq. (1), which converge to the outer attractor, are shown for $\mathrm{Bm}=4 \mathrm{G}$ and $\mathrm{fm}=240 \mathrm{~Hz}$.

set higher than we, the center peak disappeared (see the $260 \mathrm{~Hz}$ data of Fig. 2).

Figure 3 shows the phase amplitude of the outer beamlet (squares) measured with an oscilloscope and the phase amplitude obtained from the phase detector (diamonds). Note here that our phase detector was not intended for use with more than one beam bunch present. It seems that our phase detector measured the centroid of these two beamlets shown in Figs. 1 and 3. Only when the outer beamlet becomes the dominant charge distribution, was the phase detector able to measure its phase. In the lower part of Fig. 3, the phase response data from square wave dipole field modulation were shown to compare with the attractor solutions. Since the square wave can be expanded in Fourier series,

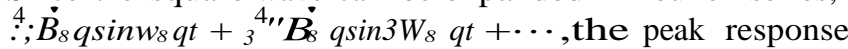
was expected and observed at a modulation frequency of 87.3 Hz.

A computer simulation based on Eq. (1) has been performed to demonstrate the coherent and incoherent nature of the single particle dynamics of the parametric resonance system. One of the results is shown in Fig. 4, where the black region corresponds to initial phase space coordinates converging toward the outer attractor. The complementary phase space coordinates converge mostly to the inner attractor except a small patch of phase space coordinates located on the boundary of the separatrix, which will converge toward two attractors located on the separatrix. The significance of these new attractors to the nonlinear dynamics is uncertain. The relative population of the inner and outer attractors observed from Fig. 2 can also be understood qualitatively from numerical simulations.
In conclusion, we have performed a first study of synchro-betatron coupling resonance in a proton storage ring. The resonance condition was created by dipole field modulation at the synchrotron frequency. The resulting beam behavior exhibited characteristics of attractors of a dissipative parametric resonant system. The transverse dipole field modulation resulted in a very unusual bunch shape distribution in the longitudinal phase space. Data observed from the oscilloscope revealed that the bunch split into two beamlets located at amplitudes corresponding to the steady state solution of the dissipative parametric resonant system. These two beamlets were observed to rotate in the synchrotron phase space at the modulation frequency. A detailed understanding of how these attractor solutions are populated is important in the study of multiparticle dynamical systems.

Our experimental result indicated that effects of ground vibration and of power supply ripple may be important through synchro-betatron coupling. Detailed data analysis based on Hamiltonian mechanics, including 6D phase space analysis, and its implication to the SSC and RHIC will be published shortly elsewhere.

This work was supported in part by a grant from National Science Foundation NSF PHY-9221402 and from the DOE DE-FG02-93ER4081.

[1] M. Ellison et al., Phys. Rev. Lett. 70, 591 (1993).

[2] L.D. Landau and E.M. Lifschitz, Mechanics (Pergamon Press, New York, 1976), 3rd ed.; D. D'Humieres, M.R. Beasley, B.A. Huberman, and Libchaber, Phys. Rev. A 26, 3483 (1982); T. Ieiri and K. Hirata, Proceedings of the IEEE Particle Accellerator Conference, Chicago (IEEE, New York, 1989), p. 926; M. Octavia, Phys. Rev. B 29, 1231 (1984).

[3] E.M. McMillan, Phys. Rev. 68, 143 (1945); V.I. Veksler, C.R. Acad. Sci. U.R.S.S., 43, 329 (1944); 44, 365 (1944).

[4] E.D. Courant and H.S. Snyder, Ann. Phys. (N.Y.) 3, 1 (1958).

[5] M.C. Crowley-Milling and 1.1. Rabinowitz, IEEE Trans. Nucl. Sci. 18, 1052 (1971); SPEAR group, ibid., 22, 1366 (1975); A. Piwinski, ibid., 24, 1408 (1977); A Piwinski, in Proceedings of 11th International Conference on High Energy Accelemtor Conf. (CERN, Geneva, 1980), p. 754; A. Piwinski, Synchro-betatron Resonances, Proceedings of the CERN Accelerator School, edited by S. Thrner (CERN Report No. 87-03, 1987), p. 187.

[6] Summary and Presentation of the Workshop on Vibmtional Control and Dynamic Alignment Issues at the SSG, edited by H.J. Weaver (Report No. SSCL-SR-1185, 1992).

[7] S.Y. Lee et al., Phys. Rev. Lett. 67, 3768 (1991); D.D. Caussyn et al., Phys. Rev. A 46, 7942 (1992).

[8) Handbook of Mathematical Functions, edited by $M$. Abramowitz and I.A. Stegun (National Bureau of Standards, 1975). 